\title{
BMJ Open Improving swallowing outcomes in patients with head and neck cancer using a theory-based pretreatment swallowing intervention package: protocol for a randomised feasibility study
}

\author{
Roganie Govender, ${ }^{1,2}$ Christina H Smith, ${ }^{3}$ Benjamin Gardner, ${ }^{2,4}$ Helen Barratt, ${ }^{5}$ \\ Stuart A Taylor 6
}

To cite: Govender R, Smith $\mathrm{CH}$, Gardner B, et al. Improving swallowing outcomes in patients with head and neck cancer using a theory-based pretreatment swallowing intervention package: protocol for a randomised feasibility study. BMJ Open 2017;7:e014167. doi:10.1136/bmjopen-2016014167

- Prepublication history for this paper is available online. To view these files please visit the journal online (http://dx.doi.org/10.1136/ bmjopen-2016-014167).

Received 9 September 2016 Revised 6 February 2017 Accepted 23 February 2017

CrossMark

For numbered affiliations see end of article.

\section{Correspondence to} Roganie Govender; Roganie.Govender@uclh.nhs. uk

\section{ABSTRACT}

Introduction: The incidence of head and neck cancer (HNC) in the UK is rising, with an average of 31 people diagnosed daily. Patients affected by HNC suffer significant short-term and long-term posttreatment morbidity as a result of dysphagia, which affects daily functioning and quality of life (QOL). Pretreatment swallowing exercises may provide additional benefit over standard rehabilitation in managing dysphagia after primary HNC treatments, but uncertainty about their effectiveness persists. This study was preceded by an intervention development phase to produce an optimised swallowing intervention package (SIP). The aim of the current study is to assess the feasibility of this new intervention and research processes within a National Health Service (NHS) setting.

Method and analysis: A two-arm non-blinded randomised controlled feasibility study will be carried out at one tertiary referral NHS centre providing specialist services in HNC. Patients newly diagnosed with stage III and IV disease undergoing planned surgery and/or chemoradiation treatments will be eligible. The SIP will be delivered pre treatment, and a range of swallowing-related and QOL measures will be collected at baseline, 1, 3 and 6 months posttreatment. Outcomes will test the feasibility of a future randomised controlled trial (RCT), detailing rate of recruitment and patient acceptance to participation and randomisation. Salient information relating to protocol implementation will be collated and study material such as the case report form will be tested. A range of candidate outcome measures will be examined for suitability in a larger RCT.

Ethics and dissemination: Ethical approval was obtained from an NHS Research Ethics Committee. Findings will be published open access in a peerreviewed journal, and presented at relevant conferences and research meetings.

Trial registration number: ISRCTN40215425; Preresults.

\section{Strengths and limitations of this study}

- Use of a randomised controlled trial design to minimise bias and differences between groups.

- Study design incorporates prior qualitative work to optimise adherence to the intervention.

- Method includes consultation with clinicians involved in usual care to devise a usual care protocol to facilitate consistency.

- Limited to one hospital site.

- Patients and clinicians are not blinded to randomisation allocation.

\section{INTRODUCTION}

\section{Background}

Incidence of head and neck cancer (HNC) in the UK is rising ${ }^{1}$ with an average of 31 people receiving a new diagnosis daily. ${ }^{2}$ This increase is primarily attributed to the rise in human papilloma virus (HPV)-related oropharyngeal cancer. ${ }^{3}$ Individuals with HPV-induced malignancy are often much younger than those with cancers induced by smoking and alcohol, and often otherwise healthy and in active employment. Reducing the morbidity of cancer treatments is a priority for the NHS Cancer Reform Strategy ${ }^{4}$ and the Macmillan Living with and Beyond Cancer Programme. ${ }^{5}$ Patients affected by HNC suffer significant short-term and longterm post-treatment morbidity as a result of dysphagia, which affects daily functioning and quality of life (QOL). Swallowing difficulties may arise secondary to the presence of tumours in the mouth and throat, ${ }^{6}$ from damage or resection of swallowing-related soft tissues and nerves during surgical intervention $^{7}$ and from the side effects and long-term tissue damage following chemoradiation. ${ }^{8}$ Swallowing interventions that may ameliorate 
the problems associated with eating and drinking after primary HNC treatments (surgery, chemoradiotherapy) are an important part of the care delivered to patients diagnosed with HNC. ${ }^{9}$

\section{Current UK practice for managing dysphagia in HNC}

Traditionally, patients would have seen a speech and language therapist (SLT) following their cancer treatment for rehabilitation of their swallowing and communication difficulties. A gradual shift in practice occurred following publication of the National Institute for Health and Care Excellence (NICE) Improving Outcomes Guidance, ${ }^{10}$ which recommend that patients should also see an SLT prior to their treatment to inform them of the likely impact to their swallowing and speech function. This information giving is also viewed as a necessary part of the process of informed consent. In support, an emerging number of functional outcome studies have indicated that swallow function pre treatment could be a strong predictor of long-term swallow function post-treatment. ${ }^{11-13}$

Implementation of the NICE recommendation is currently variable. In some centres, patients are provided with brief information by the SLT at the time of seeing their medical consultant in a multidisciplinary clinic setting. In other centres, separate SLT consultations take place, which include documentation of baseline functional measures for swallowing and communication, as well as advice on diet modification and the recommendation to start a general protocol of swallowing exercises. The latest report from the UK National Database of Head and Neck Oncology (DAHNO) ${ }^{14}$ indicated that only $29 \%$ of patients with $\mathrm{HNC}$ in England and Wales were recorded as having a pretreatment SLT consultation. Given the clinical and financial resource implications of a separate pretreatment SLT consultation, implementation will likely only increase if there is clear specification as to the optimal content of the consultation, together with evidence supporting its benefit on patients' outcomes. Aside from information giving, there is a need to define what interventions administered before treatment (prehabilitation) may improve patient experience and/or post-treatment function.

\section{The role and context of pretreatment swallowing exercises}

The physiological rationale for prophylactic swallowing exercises has been previously described in the literature. Strength-based exercises and/or range of movement exercises aimed at the swallowing musculature may prevent muscle atrophy and reduce or delay the impact of radiation-induced fibrosis. ${ }^{15-18}$ Preconditioning through exercises has been reported to be helpful in other types of surgery. ${ }^{19}$ Swallowing is described as a 'suboptimal activity ${ }^{20}$ meaning that it can be adequate for the purpose of obtaining oral nutrition even when not at maximal physiological functioning. While it seems intuitive that pretreatment swallowing exercises should be helpful in increasing physiological reserve, reducing disuse atrophy and possibly delaying the onset of fibrosis, uncertainty about its effectiveness in improving swallowing for patients with HNC persists. ${ }^{21}$ Few randomised clinical trials have attempted to address this knowledge gap, with mixed results. ${ }^{1722-28}$ Trials evaluating the effects of pretreatment swallowing exercises suffer limitations in study design, and the lack of consistent outcome measures across studies is problematic. ${ }^{21}$ Uncertainty also remains as to the optimal type and frequency of exercises although such considerations are less relevant to tailored interventions guided by prior physiological assessment. The practice of providing prophylactic swallowing exercises varies among UK clinicians, ${ }^{29}$ and to date there is no published UK data supporting the effectiveness of pretreatment swallowing exercise interventions in the HNC population. ${ }^{21}$ Any pretreatment intervention package will need to address often poor patient adherence to swallowing exercises. ${ }^{22} 24{ }^{30}$ Improved adherence may be achieved by facilitating a change in patient behaviour. A new intervention will also need to be compatible with the broader cancer care pathway as detailed in figure 1 .

\section{Development of a tailored pretreatment swallowing intervention package (SIP)}

In preparation for the feasibility study described in this manuscript, an optimised SIP was developed by the researchers, guided by the methodology proposed by the Medical Research Council (MRC) for devising complex interventions incorporating multiple components that may interact in producing outcomes. ${ }^{31}$ The scope of the current work relates to the development and feasibility testing phases of a complex intervention, but the framework also includes evaluation and implementation phases with updated guidance that incorporates a process evaluation. ${ }^{32}$ We briefly describe the development work below.

Recognising the poor patient adherence reported in previous studies of swallowing exercises, ${ }^{22} 24{ }^{30}$ we paid specific attention to optimising patient adherence during the development phase by drawing on theoretical frameworks and tools (The Behaviour Change Wheel, Behaviour Change Technique Taxonomy v1) from the field of behavioural science. ${ }^{33} 34$

The new SIP-Swallowing Intervention Package: SelfMonitoring, Assessment, Rehabilitation Training (SIP SMART) was devised using current best evidence of swallowing assessment, ${ }^{35}$ as well as insights from our earlier studies exploring the behavioural dimensions of this complex intervention; a systematic review of the literature $^{36}$ and a patient interview study (submitted manuscript).

In our systematic review of behavioural swallowing intervention studies, ${ }^{36}$ we used a published taxonomy (BCTTv1) ${ }^{33}$ of 93 hierarchically organised behaviour change techniques (BCTs), to identify the BCTs reported in the literature. BCTs may be defined as the smallest active ingredient of an intervention that may bring about a change in behaviour, for example, demonstration of the behaviour or self-monitoring of the behaviour. 


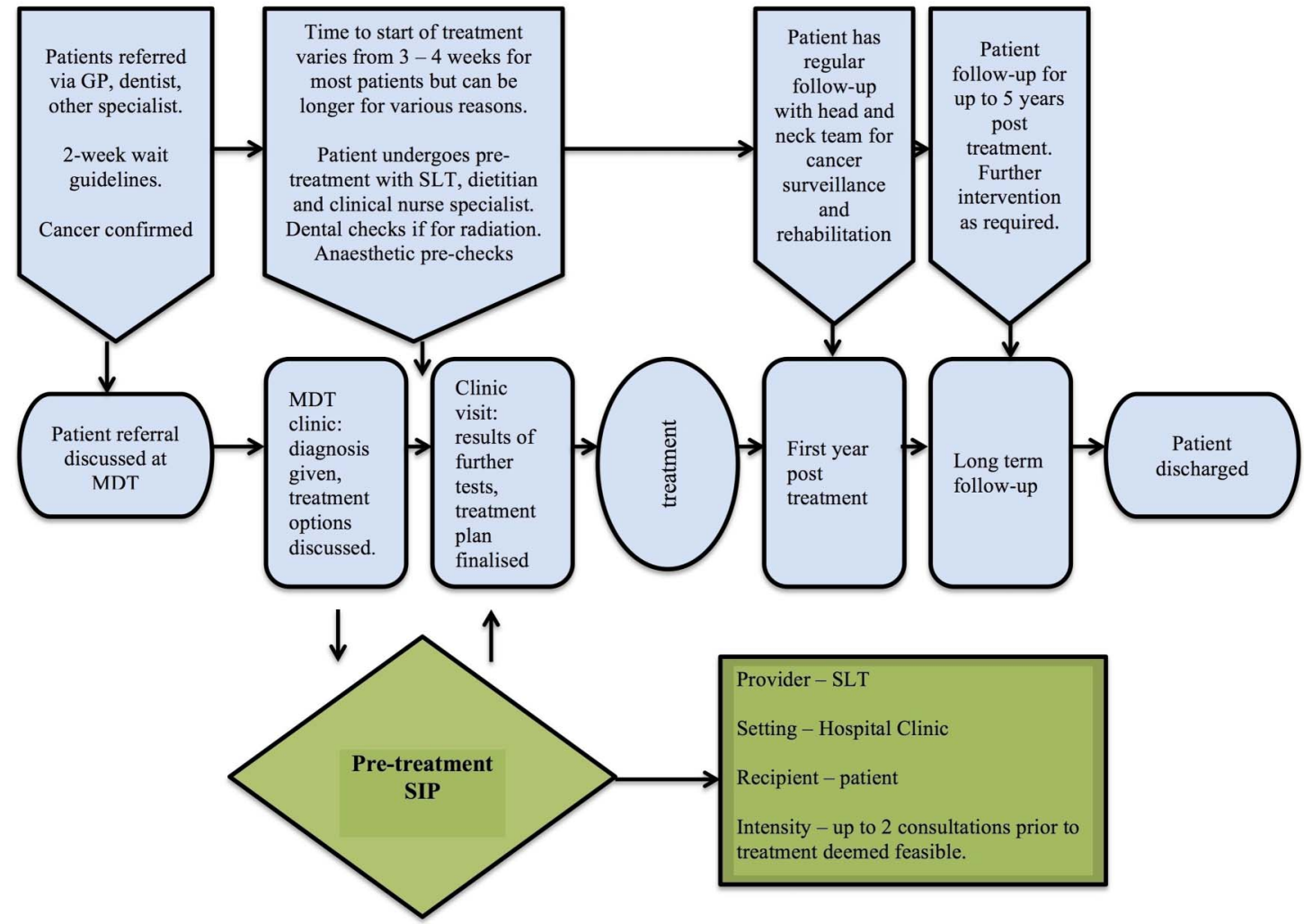

Figure 1 Pathway of care for patients with head and neck cancer.

We surmised that the BCTs that were more frequently associated with successful interventions were more likely to be successful in future interventions.

We also carried out semistructured interviews with a group of patients $(n=13)$ who had completed treatment for HNC to obtain their views and experiences of swallowing rehabilitation exercises, and in addition obtained feedback from respondents about the potential use of video animations (Dysphagia App, Northern Speech Services, USA) as an educational tool in explaining the basic mechanics of swallowing. The barriers and facilitators to exercise adherence revealed in our qualitative study informed our behavioural analysis and the subsequent selection of BCTs for the SIP SMART intervention.

We ensured that the new SIP would meet mandatory guidelines ${ }^{10}$ for information provision and informed consent, and that it could feasibly be incorporated within the existing cancer pathway for patients with HNC. The new SIP was discussed and refined by clinician (Royal College of Speech and Language Therapist expert clinician group (RCSLT) ) and patient (patientpublic involvement group (PPI)) stakeholder groups. Intervention manuals were produced for the new intervention and usual care in collaboration with the stakeholder groups. Figure 2 is a schematic diagram illustrating an overview of this process and how it links to the MRC complex interventions framework.

Although another complementary non-randomised UK trial of pretreatment swallowing intervention is planned, ${ }^{37}$ our SIP SMART trial is unique as it is based on an optimised pretreatment intervention using tailored exercises selected after physiological assessment of swallowing, and is directly informed by theoretically derived BCTs. Furthermore, comprehensive evaluation of a new SIP is likely best performed using a randomised controlled trial (RCT) design. Given the uniqueness of our SIP and proposed future RCT evaluation, a feasibility study was deemed imperative to ascertain viability within a NHS context, and to identify and address potential barriers to future roll out on a larger scale.

In particular, the aims of this feasibility study are as follows:

- Assess the rate of recruitment of eligible participants and identify any specific barriers to recruitment.

- Determine the acceptability of randomisation, and the randomisation procedure to patients and the clinical care team.

- Determine retention and attrition over the time course of the study.

- Evaluate the ease of protocol implementation, including research processes, and identify barriers in the clinical setting.

- Evaluate a range of potential outcome measures, including the ease and completeness of data collection across various time points.

- Determine concordance between potential outcome measures and define the most suitable primary outcome for the definitive study.

- Collect data to inform future sample size calculation. 


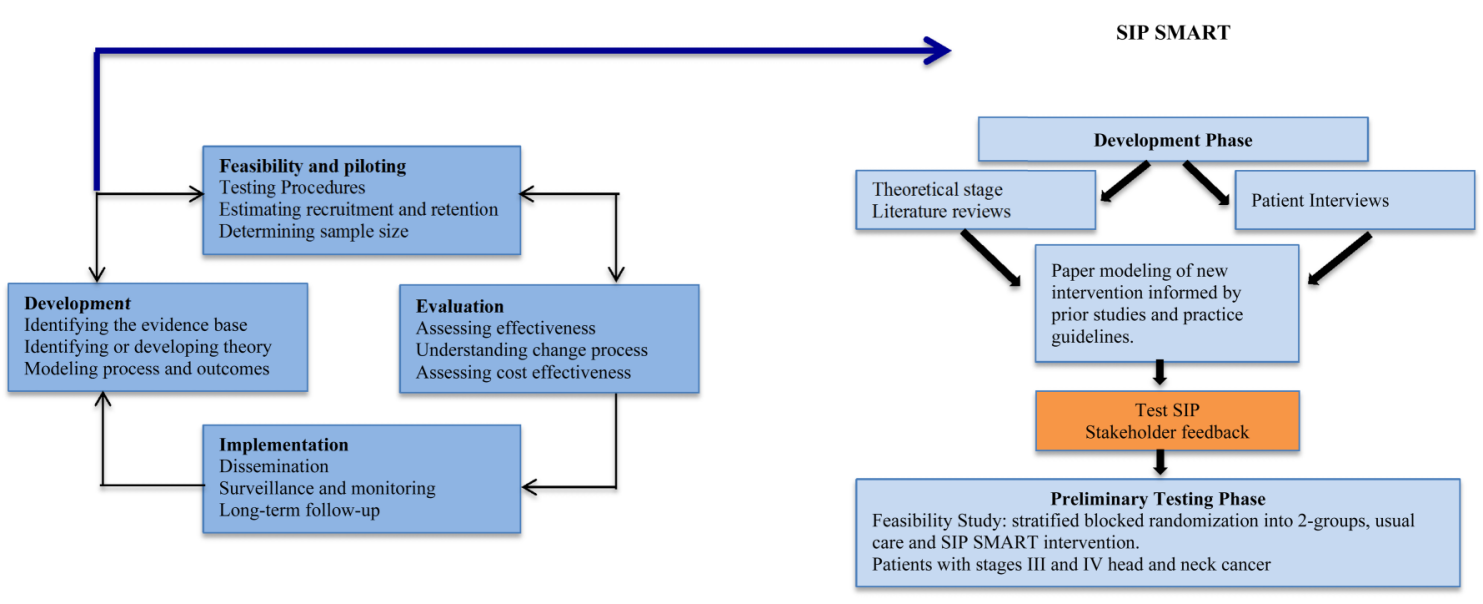

Figure 2 Key stages of the *MRC complex interventions framework that informed the development and preliminary testing of the SIP SMART intervention. *Source: Craig et al, BMJ;2008. MRC, Medical Research Council; SIP SMART, Swallowing Intervention Package: Self-Monitoring, Assessment, Rehabilitation Training.

\section{METHODS AND ANALYSIS \\ Study design}

This study forms the preliminary testing phase described within the MRC complex interventions framework. Following on from our development work, we will conduct a two-arm parallel group non-blinded randomised controlled feasibility study. The SPIRIT ${ }^{38}$ (Standard Protocol Items for Randomised Interventional Trials) checklist of was used to inform the content of this protocol.

\section{Study population, setting and recruitment plan}

The study will take place at a single NHS hospital site (Head and Neck Cancer Centre) with a catchment population of 1.5 million. The study sample will be drawn from the population of patients with newly diagnosed HNC referred to the cancer centre, and discussed at the weekly multidisciplinary team (MDT) meeting. Potential patients for the study (based on diagnosis) will be identified during the meeting by the researcher (RG), research nurse or other members of the MDT. The research team will ensure that the treating consultant is aware of potentially eligible patients so that he/she may introduce the study during the consultation with the patient if appropriate. For this feasibility study, sample size was determined pragmatically using the general guidance suggested by Lancaster and colleagues $^{39}$ who recommend that $\mathrm{n}$ of 30 is sufficient to estimate key parameters in a feasibility study. Based on a conservative annual referral of $\sim 70$ newly diagnosed stage III and stage IV patients with HNC to the Head and Neck Centre, we estimated that it will take about 9 months to recruit a total of 32 patients to this study based on recruiting $60 \%$ of eligible patients. Eligibility criteria for inclusion in the study are listed in box 1 .

\section{Prescreening/screening}

All patients who meet the clinical eligibility criteria identified at each MDT meeting will be recorded on the study screening $\log$ by the researcher or research nurse.

\section{Box 1 Inclusion and Exclusion Criteria}

Inclusion:

- Patients with newly diagnosed stage III and stage IV head and neck cancer.

- Discussed at the head and neck MDT and planned for treatment via surgery and/or chemoradiotherapy or combinations thereof.

- Able to provide informed consent.

- Proficiency in English satisfactory to participate/engage in the intervention.

- Aged 18 and above.

Exclusion:

- Patients with previous head and neck cancer treatment.

- Patients who are mid treatment or those receiving palliation.

- Patients who are to be treated solely by non-standard treatment that is not surgery, radiotherapy, chemoradiotherapy or combinations thereof. Patients treated by chemotherapy, brachytherapy and photodynamic therapy alone will be ineligible.

- Patients who are planned for a total laryngectomy.

- Patients who are considered vulnerable or unable to provide informed consent.

- Patients with brain tumours and other primary sites not within head and neck.

Screening will take place at the outpatient clinic consultation when treatment options are discussed by the surgeon/oncologist. The researcher/clinician will attend the consultations for eligible patients. If appropriate at this stage, the purpose of the study will be explained and patients will be given the patient information leaflet to take away. Most patients will be booked for repeat visits to the head and neck clinic prior to finalising their treatment plan. Due consideration will be given to ensuring that the study information is discussed at an appropriate time after the diagnosis. Patients will be given a minimum of 24 hours after receiving the patient information sheet before a mutually agreed time is arranged to 
answer any further questions to assist patients in deciding about whether to participate. The time frame was chosen because most patients return to the hospital for other tests the day after their clinic visit. This offers an opportunity to answer questions in person and obtain signed consent if appropriate. Patients will be reassured that participation is voluntary with the freedom to withdraw at any stage, and that participation in the study will not affect or delay their main treatment.

\section{Enrolment/consent}

Informed consent will be obtained by the researcher/ clinician (RG) or a trained research nurse. Following informed consent and generation of a patient study identification number, the patient will be entered onto the study enrolment $\log$ and randomised to either the SIP SMART intervention or usual care group as detailed below.

\section{Randomisation and allocation}

Eligible patients will be randomly assigned in a 1:1 ratio between usual care and intervention groups (figure 3 for trial flow chart). Patients will be stratified by first-line treatment; surgery or chemoradiation, a known factor that impacts swallowing outcomes. ${ }^{13}$ It will therefore be necessary to ensure a balance of primary treatment modality across the groups. Owing to the small numbers in this study, random block permutations will be employed to ensure a close match in numbers in the intervention and usual care groups at any given point during the trial.

Patients will be allocated to one of two groups using an online computer-generated randomisation service provided by an external company: http://www. sealedenvelope.com/. The company is registered with the Information Commissioners Office (ICO) and inspected by the MHRA (UK trials regulator). Following consent, the researcher or research nurse will enter the password-protected website and complete relevant information regarding first-line treatment. Randomisation is immediate, and the group allocation is emailed within a few minutes. This process is undertaken in the presence of the patient after signed consent is obtained, ensuring that the allocation is concealed until this point and simultaneously made known to the patient and researcher. Allocation is not blinded as the patient and staff will be aware that the new intervention includes a baseline videofluoroscopy or X-ray of swallowing. Patients allocated to the usual care group are advised that they will be sent an appointment in the post to see an SLT prior to their treatment as per the usual care pathway. Patients allocated to the intervention group are given an appointment and a further information leaflet on having a videofluoroscopy to assess how well the muscles and nerves function when swallowing different textures of food and drink. All patients are given three questionnaires to complete and return prior to their appointment with the SLT.

\section{Interventions and procedures}

Usual care group: This group will receive the usual pretreatment care offered by the SLT prior to their upcoming surgery and/or chemoradiation. The SLT clinical team consisting of four members participated in a series of consensus meetings regarding the delivery of usual care to facilitate equipoise. All four team members involved in the delivery of usual care have also undergone good clinical practice (GCP) training. A usual care manual was written and agreed by the SLT clinical team prior to initiation of the study to ensure a level of consistency among the clinicians. Usual care pretreatment is one 45 min consultation as described below:

- General case history taking and introduction of SLT role.

- Clinical baseline screening of swallowing and communication function. This is usually based on an oromotor assessment; $100 \mathrm{~mL}$ water swallow test; ${ }^{40}$ a clinician-rated Performance Status Scale indicating the normalcy of diet texture and public eating score ${ }^{41}$ and a clinician-rated scale for chewing, communication and swallowing. ${ }^{42}$ Maximal jaw opening using a TheraBite measure and voice quality ratings using the GRBAS (Grade of Hoarseness, Roughness, Breathiness, Asthenia, Strain) rating scale are also recorded.

- The patient is provided with a general overview of the planned treatment (surgery or chemoradiation) and information about the likely side effects such as mucositis and taste changes and impact of treatment on swallowing and communication function.

- General advice and exercises are offered to patients planned for chemoradiation at this appointment. Patients are provided with a general exercise sheet that includes instructions for eight different swallowing exercises, for example, passive jaw stretches. This is included as part of the information pack given to all patients receiving radiotherapy. Patients are advised that it may be helpful to start doing the exercises before treatment.

Intervention group: Patients in the intervention group will be pretreated according to the SIP SMART protocol that includes the specific components of the new intervention in addition to all aspects of usual care. The intervention takes place over two 45 min consultations that may follow each other on the same day or with a day or two between them depending on patient preference. The new intervention will be delivered by one clinician (RG) who completed a 5-day intensive training course in behaviour change (UCL Centre for Behaviour Change), supplemented by online training in coding BCTs, as well as ongoing mentorship from an expert in behaviour change. Specific details of the new intervention have not been explicitly shared with clinicians delivering usual care to minimise contamination. Broad differences include the following:

- Patients will undergo a radiological assessment of their swallow function in the fluoroscopy suite at the 


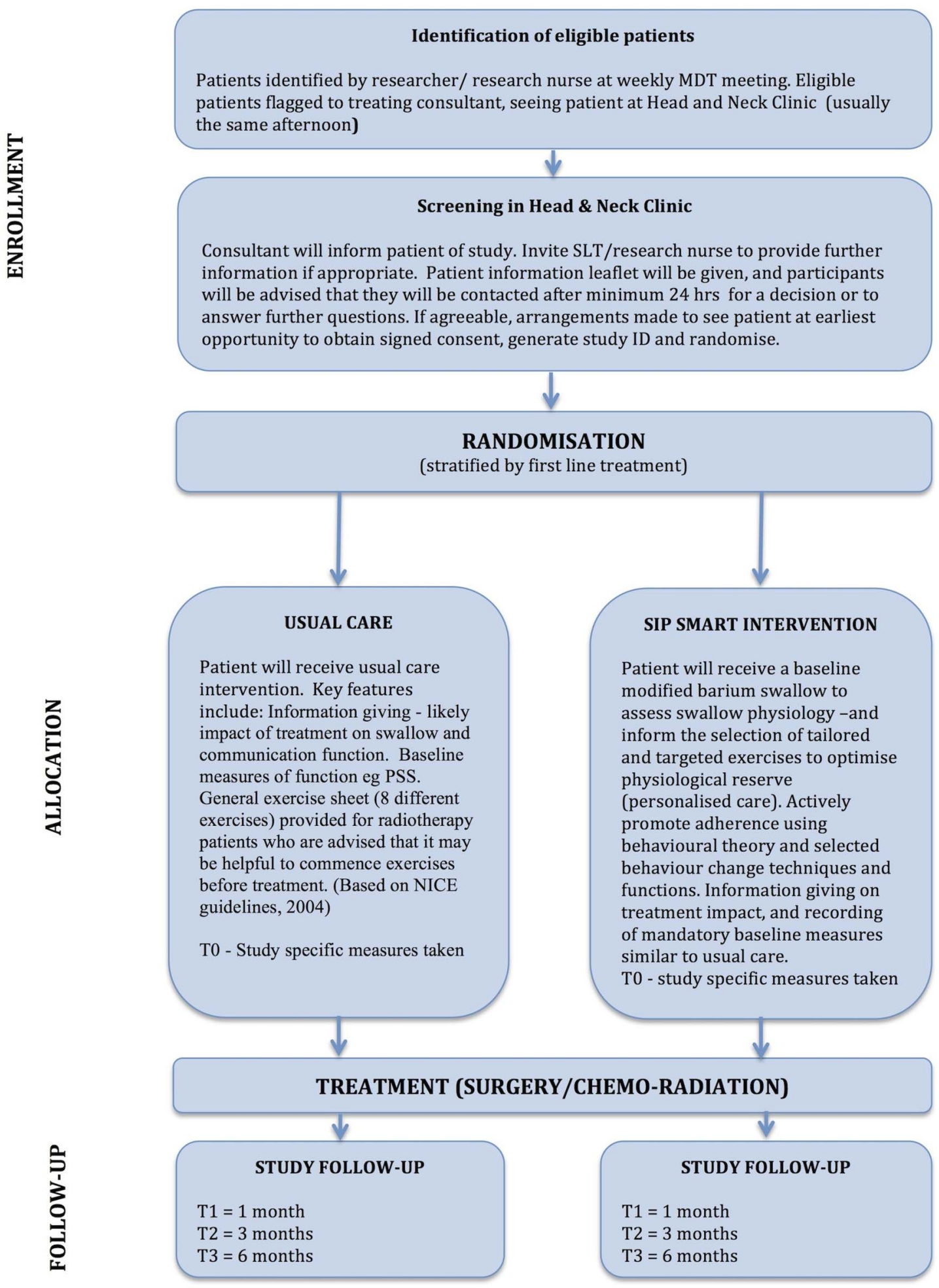

Figure 3 Trial flow chart.

hospital site. This procedure is part of the SIP SMART intervention informing the selection of targeted exercises, and is used as part of patient education. A standard protocol for this clinical procedure will be adopted for the study. ${ }^{35}$ The patient will be asked to swallow a variety of food textures; fluoroscopic images of the lateral and anterior-posterior plane captured at a rate of 30 frames per second will be recorded on a swallow workstation (Digital Kay Pentax Swallow workstation, USA). This will be available for later analysis of swallow physiology. The fluoroscopy screening time is usually $2-3 \mathrm{~min}$.

- Patients will subsequently be shown a video animation (Dysphagia App, Northern Speech Services, USA) to 
Table 1 Outcome measures and time points

\begin{tabular}{|c|c|c|c|c|}
\hline Measure & $\begin{array}{l}\text { T0 } \\
\text { baseline }\end{array}$ & $\begin{array}{l}\text { T1 } \\
1 \text { month }\end{array}$ & $\begin{array}{l}\text { T2 } \\
3 \text { months }\end{array}$ & $\begin{array}{l}\text { T3 } \\
6 \text { months }\end{array}$ \\
\hline Background information & $\mathrm{x}$ & & & \\
\hline \multicolumn{5}{|l|}{ Measures taken as part of usual care } \\
\hline Performance Status Scale (PSS) & $\mathrm{x}$ & $\mathrm{X}$ & $\mathrm{X}$ & $\mathrm{x}$ \\
\hline Maximal incisor opening (mouth opening) & $\mathrm{x}$ & $\mathrm{x}$ & $\mathrm{X}$ & $\mathrm{x}$ \\
\hline Functional Intraoral Glasgow Scale & $\mathrm{x}$ & $\mathrm{x}$ & $\mathrm{x}$ & $\mathrm{x}$ \\
\hline $100 \mathrm{~mL}$ Water swallow test & $\mathrm{x}$ & $\mathrm{x}$ & $\mathrm{x}$ & $\mathrm{x}$ \\
\hline \multicolumn{5}{|l|}{ Additional measures for trial } \\
\hline MD Anderson Dysphagia Inventory & $\mathrm{x}$ & $\mathrm{x}$ & $\mathrm{x}$ & $\mathrm{X}$ \\
\hline General Self-Efficacy Scale & $\mathrm{x}$ & & & $\mathrm{x}$ \\
\hline Self-reported adherence question & & $\mathrm{x}$ & $\mathrm{x}$ & $\mathrm{x}$ \\
\hline HRQOL-FACT-HN & $\mathrm{x}$ & $\mathrm{x}$ & $\mathrm{x}$ & $\mathrm{x}$ \\
\hline Modified Barium Swallow Impairment Score and & & & & $\mathrm{x}$ \\
\hline Penetration/Aspiration score & & & & \\
\hline Acceptability to participation/randomisation questionnaire & & & & $\mathrm{x}$ \\
\hline
\end{tabular}

explain the basic mechanics of swallowing and to orientate them to key structures such as the tongue, base of tongue, airway and oesophagus. Patients will thereafter be shown their own videofluoroscopy and helped to identify the key structures using this newly acquired knowledge. The clinician will encourage the patient to provide commentary and or ask questions as they watch their own swallowing.

- The videofluoroscopy assessment will be used to tailor the information, advice and exercises given to the patient during the pretreatment session and to facilitate discussion about the rationale for exercises and possible consequences of not doing exercises.

- Further detail about the intervention content and behavioural strategies used is provided in the SIP SMART manual. This is not included in this paper, but can be requested from the first author.

Patients in both groups will follow the usual care pathway for SLT rehabilitation post treatment (see figure 3 for trial flow chart). The number of SLT rehabilitation sessions for all patients will be recorded. Patients will be informed that exercises may be amended post treatment based on updated swallowing assessment.

\section{Baseline and follow-up outcome measures}

Swallowing is a multidimensional phenomenon that may be measured by a number of different indicators including: patient-reported outcome measures, clinician ratings and scores from instrumental assessments such as videofluoroscopy and fibre-optic endoscopic evaluation of swallowing. This range of outcomes can prove problematic when synthesising findings from multiple studies. In this feasibility trial, we have selected a similar panel of swallowing outcome measures to that used in concurrent UK trials for HNC (radiation and surgery-based trials using a swallowing outcome measure) ${ }^{43}$ as well as measures collected as part of routine clinical practice (table 1). Outcome measures will be collected at baseline and 1,3 and 6 months after treatment. Six months represents a relatively stable time point in the recovery trajectory for patients with $\mathrm{HNC}$ and was therefore selected as an appropriate end point. ${ }^{21} 36$ Patient weight, body mass index and use of feeding tube will also be recorded at all time intervals. The MD Anderson Dysphagia Inventory (MDADI ${ }^{44}$ will be used to determine swallowing related QOL. We have chosen to collect information on health-related QOL via the Functional Assessment of Cancer Therapy and Head and Neck subscale (FACT-HN) as this questionnaire was identified as the most preferred by patients with HNC when compared with other QOL questionnaires. ${ }^{45}$

At 6 months, a videofluoroscopy will be conducted on all patients using the standardised Modified Barium Swallow Impairment Profile Protocol (MBS Imp Profile), and analysis. ${ }^{35}$ Three SLT clinicians who have completed a 25 hour online training module and obtained the minimum $80 \%$ reliability score will provide consensus ratings for these assessments. Standard assessment rating forms developed as part of the MBS Imp Profile will be used to score the videofluoroscopies. We will also rate aspiration from the videofluoroscopies, based on the widely used 8-point Penetration-Aspiration Scale (PAS) ${ }^{46}$

As we anticipate that adherence to swallowing exercises should be associated with better swallowing, we will also collect intermediate outcomes on adherence using two simple questions developed for this trial. The questions will ask about percentage adherence over a specified time, and a free text question to gather further information about any specific reasons for adherence/ non-adherence to exercises.

\section{Safety considerations}

We do not anticipate any serious safety concerns arising from this largely behavioural intervention. The use of the videofluoroscopy as part of the intervention is sometimes associated with aspiration of barium contrast material but barium pneumonitis is reported to be rare 
at $<1 \%{ }^{47}$ The additional radiation exposure associated with the procedure is roughly the equivalent of 2 months of background radiation that an adult in the UK may experience from the environment, and considered minor in the context of the patient's overall treatment. The procedure will be undertaken by an experienced SLT familiar with the protocol for dealing with an adverse event related to barium inhalation. The procedure is also a well-established part of routine clinical practice.

\section{Data collection and management}

We devised a number of study specific forms including a case report form (CRF), screening log, enrolment log, training $\log$ for any study-related training and file note entry forms for any relevant ad hoc communication. Patient names will be replaced by a study number on all study forms and completion of CRFs will be in accordance with GCP guidelines. A site file containing all relevant documents as stipulated by local research and development and governance guidelines will be maintained throughout the study and securely stored in a locked cabinet. All patient-reported questionnaires will also be securely filed. Non-identifiable quantitative data will be transferred from the questionnaires and CRFs to a specifically designed Microsoft Access database by a data manager. The database will first be tested using mock data to ensure that it meets the requirements for data entry. A random check of $\sim 10 \%$ of the data inputted will be reviewed against the original source by the researcher (RG) to estimate an error rate. This information will help ensure that a robust data management plan is in place for a more formal trial. The researcher will maintain an electronic diary of relevant information pertaining to the study processes on a passwordprotected laptop computer.

\section{Analysis}

We will use mainly descriptive analysis and summary statistics to address our aims. Study screening and enrolment $\operatorname{logs}$ will be used in determining the rate of recruitment into the study. All qualitative information (researcher diary), minutes of study-related discussions and meetings will be imported into NVIVO 10, a software database to facilitate the organisation and thematic coding of qualitative or textual data. The researcher is in a unique position of being embedded within the clinical team, and therefore able to make observations over the duration of the study in a naturalistic manner. This approach to process analysis arguably may provide more useful information than the use of post hoc focus groups and interviews that rely on participant memory and may be removed from context. ${ }^{48}$ Observing and collecting information in this way also means that the researcher can observe the interplay of other factors (eg, multiple studies competing for the same patient group, prevailing views of the treating consultant about the value of the proposed intervention and how busy the clinic is) that may reveal vital information about the barriers and facilitators to recruitment.

We will also report on the practicality of implementing the protocol, for example, obtaining timeslots for videofluoroscopy; average time taken for the recruitment and consent process; utility of the chosen randomisation method and suitability of the study-specific forms including the CRF. This information will be used to optimise components of the protocol and study process in preparation for a larger trial.

As a range of outcome measures will be collected, we will look at the suitability of each measure, the ease of collection and the quality and completeness of the data collection. We will observe the relationships (concordance and discordance) between the different outcome measures to help inform the most suitable choice of primary and secondary measures for a definitive trial. Important parameters such as SD and estimates of effect size will be used to inform the sample size calculation. Based on the available literature, we will aim to specify the target difference (clinically meaningful difference) for the chosen primary outcome. The primary outcome measure will be chosen from potential candidate measures on the basis that it is valid, practical and feasible to obtain and has expert agreement (RCSLT clinical expert group) that it reflects a good summary measure to answer the question of whether the new intervention is effective in improving swallowing.

Patient acceptability to participation and randomisation will be determined using a previously developed questionnaire. $^{49}$ Self-reported adherence to the intervention will be explored via a brief study questionnaire. Previous studies reported that full adherence to exercises during radiotherapy was under $15 \% .{ }^{27}{ }^{30}$ In a Danish study of a similar intervention to the current study, an average of $35 \%$ of patients reported doing their exercises at least once a day between 1 month and 11 months after treatment. ${ }^{22}$ We have therefore selected $35 \%$ as the minimum target adherence for our study.

\section{Criteria for success}

This study will be viewed as feasible to proceed to a definitive trial if:

- a suitable outcome measure is determined, and sample size estimated;

- recruitment rate into the trial reaches an average of four patients a month;

- patients report generally positive views about participation and acceptance to randomisation as determined by questionnaire evaluation;

- patients in the intervention group are more adherent than those in usual care, with at least $35 \%$ of intervention group patients reporting satisfactory to good adherence to exercises.

\section{DISCUSSION}

To the best of our knowledge, this is the first randomised UK study of a behavioural swallowing exercise 
intervention for patients with HNC registered on the trials database. By undertaking a feasibility study and identifying key uncertainties, any future study may be optimised to make best use of resources. ${ }^{50}$ This study follows earlier work on intervention development bringing together expertise from different fields including clinical dysphagia management and behaviour change. It benefits from the use of newer paradigms in health research including the use of consultative and consensus meetings to devise a treatment manual to specify the content of usual care, a common omission when reporting such interventions. ${ }^{51}$ It is likely to provide a rich source of information about how readily patients with a new diagnosis of HNC will accept and participate in a self-management-type intervention. It will also provide a preliminary indication of the recruitment potential for rehabilitation therapy trials for this population. A recent UK study ${ }^{52}$ that randomised HNC patients to either a pretreatment gastrostomy tube or a nasogastric tube reported recruiting only $23 \%$ of eligible patients, highlighting the importance of this feasibility work. It is well known that the treatment of HNC involves a complex care pathway with multiple disciplines being involved, particularly at the pretreatment stage. The feasibility of undertaking a clinical trial at this point in the patient pathway is compounded by the challenges of approaching patients to participate in a trial shortly after receiving a cancer diagnosis. Insights from this study may therefore have more widespread relevance for future studies of this population.

Limitations of the study include the inability to blind participants and staff to the randomisation allocation as all patients in the intervention group will receive a videofluoroscopy as part of the intervention. Based on the limited number of individuals trained in rating videofluoroscopies using the MBS impairment profile, the same SLTs involved in usual care delivery will be rating the assessments. For this reason, we have chosen to use a consensus rating from three clinicians as this method is likely to introduce the least bias. We have not planned to evaluate fidelity in delivering the intervention at this stage, as only one individual will be delivering the intervention (RG). In a larger trial, training will be required by all clinicians before delivering the intervention and fidelity checks will be built into the research process. In spite of these limitations, the current study represents a first and important step towards examining the feasibility of undertaking a fullscale RCT within NHS hospitals to determine the effectiveness of a pretreatment swallowing intervention for patients with HNC, delivered by SLTs.

\footnotetext{
Author affiliations

${ }^{1}$ University College London Hospital, Head \& Neck Cancer Centre, London, UK

${ }^{2}$ Department of Behavioural Science \& Health, University College London, London, UK

${ }^{3}$ Division of Psychology \& Language Sciences, University College London, London, UK

${ }^{4}$ Department of Psychology, Institute of Psychiatry, Psychology and

Neuroscience (IoPPN), Kings College London, London, UK
}

${ }^{5}$ Department of Applied Health Research, NIHR CLAHRC North Thames, University College London, London, UK

${ }^{6}$ Centre for Medical Imaging, University College London, London, UK

Acknowledgements The authors would like to thank the patients and clinicians who participated in the development of the SIP SMART intervention, as well as all members of the UCLH Head and Neck MDT for their support with this study. We are grateful for the assistance of the research support team, in particular the research nurse and research support practitioner. The authors also wish to acknowledge the contribution of Professor Jane Wardle during the early planning stages of this study. Jane Wardle passed away on 20 October 2015.

Contributors RG provided the original study concept and drafted the manuscript. CHS, BG and SAT contributed to refining the study design. SAT is the chief investigator. CHS, BG, HB and SAT provided critical feedback in revising the manuscript. $R G$ is the lead researcher. All authors approved of the final manuscript.

Funding This report is an independent research supported by the National Institute for Health Research (NIHR/HEE Clinical Doctoral Research Fellowship, RG, CDRF- 2013-04-020). HB is supported by the NIHR Collaboration for Leadership in Applied Health Research and Care (CLAHRC) North Thames at Bart's Health NHS Trust. SAT is an NIHR senior investigator supported by the UCLH Biomedical Research Centre. The study is sponsored by University College London.

Disclaimer The views expressed are those of the author(s) and not necessarily those of the NHS, the NIHR or the Department of Health.

\section{Competing interests None declared.}

Ethics approval This manuscript is based on Protocol V.1.5 dated 03.02.16 - submitted and approved by the London South East NHS Research Ethics Committee (14.L0/1152).

Dissemination A lay summary of the study is available on the CRUK website. Final results of this feasibility study will be publicly available through open access publication in a peer-reviewed journal, and presented at relevant conferences and research meetings.

Provenance and peer review Not commissioned; externally peer reviewed.

Data sharing statement The intervention manual will be available from the first author on completion of the study.

Trial status Ongoing data collection.

Open Access This is an Open Access article distributed in accordance with the terms of the Creative Commons Attribution (CC BY 4.0) license, which permits others to distribute, remix, adapt and build upon this work, for commercial use, provided the original work is properly cited. See: http:// creativecommons.org/licenses/by/4.0/

\section{REFERENCES}

1. http://www.cancerresearchuk.org/health-professional/cancerstatistics/statistics-by-cancer-type/oral-cancer/incidence (accessed Aug 2016).

2. http://www.macmillan.org.uk/documents/aboutus/research/ richpictures/update/rp-people-with-head-and-neck-cancer (accessed Aug 2016).

3. Chaturvedi AK, Anderson WF, Lortet-Tieulent J, et al. Worldwide trends in incidence rates for oral cavity and oropharyngeal cancers. $J$ Clin Oncol 2013;31:4550-9.

4. http://www.nhs.uk/NHSEngland/NSF/Documents/Cancer\%20Reform \%20Strategy.pdf (accessed Aug 2016).

5. http://www.macmillan.org.uk/aboutus/healthandsocialcareprofessionals/ macmillansprogrammesandservices/survivorship.aspx (accessed Aug 2016).

6. Starmer H, Gourin C, Lua LL, et al. Pretreatment swallowing assessment in head and neck cancer patients. Laryngoscope 2011;121:1208-11.

7. Kreeft AM, Van Der Molen L, Hilgers FJ, et al. Speech and swallowing after surgical treatment of advanced oral and oropharyngeal carcinoma: a systematic review of the literature. Eur Arch Oto Rhino Laryngology 2009;266:1687-98. 
8. Wall LR, Ward EC, Cartmill B, et al. Physiological changes to the swallowing mechanism following (chemo) radiotherapy for head and neck cancer: a systematic review. Dysphagia 2013;28:481-93.

9. National Cancer Action Team-Rehabilitation Advisory Board. http:// webarchive.nationalarchives.gov.uk/20130513211237/http:/www. ncat.nhs.uk/sites/default/files/workdocs/NCAT_Rehab_ EvidenceReview 2012FINAL24 1 12.pdf

10. National Institute of Clinical Effectiveness. Improving outcomes in head and neck cancers. The Manual, 2004.

11. Van der Molen L, van Rossum MA, Ackerstaff AH, et al. Pretreatment organ function in patients with advanced head and neck cancer: clinical outcome measures and patients' views. $B M C$ Ear Nose Throat Disord 2009;9:10.

12. Wilson JA, Carding PN, Patterson JM. Dysphagia after nonsurgical head and neck cancer treatment: patients' perspectives. Otolaryngol Head Neck Surg 2011;145:767-71.

13. Kalavrezos N, Cotrufo S, Govender R, et al. Factors affecting swallow outcome following treatment for advanced oral and oropharyngeal malignancies. Head Neck 2014;36:47-54.

14. Data for Head and Heck Oncology (DAHNO). Tenth annual report. http://digital.nhs.uk/catalogue/PUB18081/clin-audi-supp-prog-headneck-dahn-13-14.pdf (accessed Aug 2016).

15. Duarte VM, Chhetri DK, Liu YF, et al. Swallow preservation exercises during chemoradiation therapy maintains swallow function. Otolaryngol Head Neck Surg 2013;149:878-84

16. Hutcheson KA, Bhayani MK, Beadle BM, et al. Use it or lose it: eat and exercise during radiotherapy or chemoradiotherapy for pharyngeal cancers. JAMA Otolaryngol Head Neck Surg 2013;139:1127-34.

17. Carnaby-Mann G, Crary MA, Schmalfuss I, et al. 'Pharyngocise': randomized controlled trial of preventative exercises to maintain muscle structure and swallowing function during head-and-neck chemoradiotherapy. Int J Radiat Oncol Biol Phys 2012;83:210-9.

18. King SN, Dunlap NE, Tennant PA, et al. Pathophysiology of radiation-induced dysphagia in head and neck cancer. Dysphagia 2016;31:339-51.

19. Jensen BT, Laustsen S, Jensen JB, et al. Exercise-based pre-habilitation is feasible and effective in radical cystectomy pathways-secondary results from a randomized controlled trial. Support Care Cancer 2016;24:3325-31.

20. Burkhead LM, Sapienza CM, Rosenbek JC. Strength-training exercise in dysphagia rehabilitation: principles, procedures, and directions for future research. Dysphagia 2007;22:251-65.

21. Perry A, Lee SH, Cotton S, et al. Therapeutic exercises for affecting post-treatment swallowing in people treated for advanced-stage head and neck cancers. Cochrane Database Syst Rev 2016;(8): CD011112.

22. Mortensen HR, Jensen K, Aksglæde K, et al. Prophylactic swallowing exercises in head and neck cancer radiotherapy. Dysphagia 2015;30:304-14.

23. Van Den Berg MG, Kalf JG, Hendriks JC, et al. Normalcy of food intake in head and neck cancer patients supported by dietary counseling and swallowing therapy: a Randomised Clinical Trial. Head Neck 2016;38(Suppl 1):E198-206.

24. Lazarus CL, Husaini H, Falciglia D, et al. Effects of exercise on swallowing and tongue strength in patients with oral and oropharyngeal cancer treated with primary radiotherapy with or without chemotherapy. Int J Oral Maxillofac Surg 2014;43:523-30.

25. Kotz T, Federman AD, Kao J, et al. Prophylactic swallowing exercises in patients with head and neck cancer undergoing chemoradiation: a randomized trial. Arch Otolaryngol Head Neck Surg 2012;138:376-82

26. Tang $Y$, Shen $Q$, Wang $Y$, et al. A randomized prospective study of rehabilitation therapy in the treatment of radiation-induced dysphagia and trismus. Strahlenther Onkol 2011;187:39-44.

27. Van der Molen L, van Rossum MA, Burkhead LM, et al. A randomized preventive rehabilitation trial in advanced head and neck cancer patients treated with chemoradiotherapy: feasibility, compliance, and short-term effects. Dysphagia 2011;26:155-70.

28. Logemann JA. An RCT comparing the shaker exercise with traditional therapy: a preliminary study. Dysphagia 2009;24:403-11.

29. Roe JWG, Carding PN, Rhys-evans PH, et al. Assessment and management of dysphagia in patients with head and neck cancer who receive radiotherapy in the United Kingdom-a web-based survey. Oral Oncol 2012;48:343-8.
30. Shinn EH, Basen-Engquist K, Baum G, et al. Adherence to preventive exercises and self-reported swallowing outcomes in post-radiation head and neck cancer patients. Head Neck 2013;35:1707-12.

31. Craig P, Dieppe $\mathrm{P}$, Macintyre $\mathrm{S}$, et al. Developing and evaluating complex interventions : the new Medical Research Council guidance. BMJ 2008;337:979-83.

32. Moore GF, Audrey S, Barker M, et al. Process evaluation of complex interventions: Medical Research Council guidance. BMJ 2015;350: h1258-h1258.

33. Michie S, van Stralen MM, West R. The behaviour change wheel: a new method for characterising and designing behaviour change interventions. Implement Sci 2011;6:42.

34. Michie S, Richardson M, Johnston M, et al. The behavior change technique taxonomy (v1) of 93 hierarchically clustered techniques: building an international consensus for the reporting of behavior change interventions. Ann Behav Med 2013;46:81-95.

35. Martin-Harris B, Brodsky MB, Michel $\mathrm{Y}$, et al. MBS measurement tool for swallow impairment--MBSImp: establishing a standard. Dysphagia 2008;23:392-405.

36. Govender R, Smith $\mathrm{CH}$, Taylor SA, et al. Swallowing interventions for the treatment of dysphagia after head and neck cancer: a systematic review of behavioural strategies used to promote patient adherence to swallowing exercises. BMC Cancer 2017;17:43.

37. Wells M, King E, Toft K, et al. Development and feasibility of a Swallowing intervention Package (SiP) for patients receiving radiotherapy treatment for head and neck cancer-the SiP study protocol. Pilot Feasibility Stud 2016;2:40.

38. Chan AW, Tetzlaff JM, Gøtzsche PC, et al. SPIRIT 2013 explanation and elaboration: guidance for protocols of clinical trials. BMJ 2013;346:e7586.

39. Lancaster GA, Dodd S, Williamson PR. Design and analysis of pilot studies: recommendations for good practice. J Eval Clin Pract 2004; 10:307-12.

40. Patterson JM, Hildreth A, Mccoll E, et al. The clinical application of the $100 \mathrm{~mL}$ water swallow test in head and neck cancer. Oral Oncol 2011;47:180-4.

41. List MA, Ritter-Sterr C, Lansky SB. A performance status scale for head and neck cancer patients. Cancer 1990;66:564-9.

42. Nicoletti G, Soutar DS, Jackson MS, et al. Chewing and swallowing after surgical treatment for oral cancer: functional evaluation in 196 selected cases. Plast Reconstr Surg 2004;114:329-38.

43. Owadally W, Hurt C, Timmins $\mathrm{H}$, et al. PATHOS: a phase II/III trial of risk-stratified, reduced intensity adjuvant treatment in patients undergoing transoral surgery for Human papillomavirus (HPV) positive oropharyngeal cancer. BMC Cancer 2015;15:602.

44. Chen AY, Frankowski R, Bishop-leone J. The development and validation of a dysphagia-specific quality-of-life questionnaire for patients with head and neck cancer: the M. D. Anderson dysphagia inventory. Arch Otolaryngol Head Neck Surg 2001;127:870-6.

45. Mehanna HM, Morton RP, Mehanna HM, et al. Patients' views on the utility of quality of life questionnaires in head and neck cancer: a randomised trial. Clin Otolaryngol 2006;31:310-16.

46. Rosenbek JC, Robbins JA, Roecker EB, et al. A penetrationaspiration scale. Dysphagia 1996;11:93-8.

47. Jo H, Park JG, Min D, et al. Incidence of pneumonia after videofluoroscopic swallowing study and associated factors. Dysphagia 2016;31:41-8.

48. Morgan-Trimmer S, Wood F. Ethnographic methods for process evaluations of complex health behaviour interventions. Trials 2016;17:232.

49. Jenkins V, Fallowfield L. Reasons for accepting or declining to participate in randomized clinical trials for cancer therapy. $\mathrm{Br}$ J Cancer 2000;82:1783-8.

50. Levati S, Campbell P, Frost R, et al. Optimisation of complex health interventions prior to a randomised controlled trial: a scoping review of strategies used. Pilot Feasibility Stud 2016;2:17.

51. Abraham C, Johnson BT, de Bruin M, et al. Enhancing reporting of behavior change intervention evaluations. J Acquir Immune Defic Syndr 2014;66(Suppl 3):S293-9.

52. Paleri V, Wood J, Patterson J, et al. A feasibility study incorporating a pilot randomised controlled trial of oral feeding plus pre-treatment gastrostomy tube versus oral feeding plus as-needed nasogastric tube feeding in patients undergoing chemoradiation for head and neck cancer (TUBE trial). Pilot Feasibility Stud 2016;2:29. 